Aus dem Neurologischen Institut zu Frankfurt a. M.

\title{
Untersuchungen über den Bau und die Innervierung des Dentins.
}

\author{
Von
}

Dr. phil. C. Fritsch, Zahnarzt.

Hierzu Tafel XIII und XIV.

Es gibt wohl wenig Fragen der normalen Histologie, die so viel umstritten und trotzdem immer noch keiner endgültigen Lösung entgegengebracht sind, als die des feineren Baues des Dentins und seiner Innervierung. Es sei mir daher gestattet, im folgenden über Untersuchungen, die ich seit März 1912 in dieser Richtung mache, zu berichten.

\section{Material und Methode.}

Als Material für die Untersuchungen wurden durchweg intakte, d. h. keine pathologisch veränderten Zähne von Homo sapiens, sowie auch von Säugetieren (Kalb, Hund, Igel) benutzt. Bei den ersteren wurde Wert darauf gelegt, solche junger Individuen, die man ja gelegentlich bei Stellungsanomalien extrahieren muss, zu verarbeiten. Bei den Tierpräparaten zeigte sich, dass die Zähne des Igels besonders vorteilhafte Bilder gaben.

Das gesamte Material wurde in Formol konserviert, was für die anzuwendenden Silberfärbemethoden zwecks Darstellung der Nerven erforderlich war (mindestens 4 Wochen). Sodann wurden die Zähne entkalkt nach der von $\mathrm{Sch}$ a ffer emptohlenen Methode, über die ausführlich $R$ eich berichtet. sowie vor allem eine weitere genaue Darstellung für die Herstellung von Gefrierschnitten gibt, denn nur solche konnten in Anwendung kommen, da der Hauptwert darauf za legen war, das Dentin mit dem Pulpengewebe zasammen als dünnen Schnitt zu erhalten. Technisch ist dies insofern schwierig, als bei der Weiterbehandlung der Schnitte, und es sei vorausbemerkt, dass sehr komplizierte Färbemethoden angewandt werden mussten, leicht das zarte Pulpengewebe sich von dem Dentin, mit dem es doch nur durch feine protoplasmatische Fortsätze verbunden ist, losreisst, und natiirlich ist so der Prozentsatz der wirklich brauchbaren Präparate kein günstiger.

Dazu kommen nun noch, wenn man so sagen darf, die launischen Färbemetboden, denn das sind alle Silbermethoden mehr oder weniger und solche kamen meist zur Anwendung.

Selbstverständlich sind auch alle anderen gebräuchlichen Färbemethoden versucht worden, zumal um den normalen Aufbau des Dentins zu studieren, da es sich dabei meist um eine elektive Färbung von bestimmten Bindegewebsfasern und protoplasmatischen Fortsätzen handelte.

Archiv f. mikr. Anat. Bd. 84. Abt. I. 
Als spezitische Nervenfärbungen wurden versucht eine neue Methode nach Münch (Methylenblan), ferner die Cajal sche und Bielschowskysche Silbermethode.

Letztere gab mit einigen Modifikationen die besten Resultate; so dass dieselbe hier nochmals ausführlich angeführt sei: Die Schnitte kommen von Aqua dest. auf 24 Stunden in reines Pyridin. Danach gründliches Auswaschen in Aqua dest., um Niederschläge zu vermeiden, am besten 24 Stunden mit öfterem Wechsel des Aqua dest. Sodann werden die Schnitte auf $5-8$ Tage in 3--5 proz. Argentum nitricum gebracht (im Dunklen aufzubewahren) und dann nach kurzem Abspülen in folgender Silberoxydammoniaklösung gefärbt. Zu $5 \mathrm{ccm} 20$ proz. Argentum nitricum fügt man 5 Tropfen 40 proz. Natronlauge; es erfolgt ein dunkler Niederschlag, der durch tropfenweises Zusetzen von reinem Ammoniak zur Klärung gebracht wird (gut umschütteln, auf jeden Fall darf kein Ammoniaküberschuss vorhanden sein).

Diese ammoniakalische Silberlösung wird dann mit Aqua dest. aufs vierfache Volumen gebracht and in diese Lösung kommen die Schnitte zuerst $4-5$ Minuten. Danach in angesüuertes Aqua dest. (1 Tropfen Eisessig) nach Abspülen in 20 proz. Formol zur Redulition. Nunmehr gründliches Auswaschen in Aqua dest. und die Prozedur von vorne beginnen; jedoch immer kiïrzer im ammoniakalischen Silber lassen. Die besten Resultate wurden trzielt nach 10-12 maligem Wiederholen dieser Färbung.

Die Schnitte wurden dann des besseren Aufbewahrens halber vergoldet und neuerdings mittels Gelatine zugedeckt. $\mathrm{Da}$ sich gerade diese neue Zudeckmethode auch für Zahnpräparate sehr gut bewährt, abgesehen von ihrer wesentlichen Ersparnis (kein Verbrauch von Alkohol, Xylol und Deckgläser), durch den Umstand, dass es einem besser gelingt, das Pulpengewebe nit dem Dentin, soweit es noch nach diesen komplizierten Färbemethoden zusammenhängt, zu erhalten; so seien kurz noch einige Worte üher diese Methode gesagt: Die Schnitte kommen vom Wasser in eine Gelatinelösung, ron da direkt auf den Objektträger und werden dann nochmals mit dieser Gelatinelösung übergossen. Die Gelatinelösung wird hergestellt ans reiner Gelatine, indem man $10 \mathrm{gr}$ derselben mit 100 Aqua dest. zum Quellen bringt, sodann im Wasserbade bei etwa $50^{\circ}$ verflüssigt, die Lösung im Brutofen filtriert. Diese Gelatinelösung hält sich allerdings nur 1-2 Tage. Die übergossenen.Objektträger lässt man 24 Stunden trocknen und erhält dann ganz einwandfreie Präparate, die man sehr gut mit ölimmersion betrachten kann.

Wenn auch aus diesen Ausführungen hervorgeht, dass für die Herstellung der Präparate grosse technische Schwierigleiten vorhanden sind und die Prozentzahl der brauchbaren Prüparate eine sehr schlechte ist, so muss doch hervorgehoben werden, dass wenn aber auch das Präparat gelingt, die Methode so Vorzügliches leistet, dass ich dieselbe nicht missen möchte, denn ein Zweifel über die Natur einer Faser kann dann nicht entstehen, da die Nervenfaser sich als feiner, scharf markierter, glattrandiger, schwarzer Faden gegenüber den Bindegewebsfasern abhebt. Gerade dieser Umstand ist, wie die späteren Ausführungen noch zeigen werden, von sehr grosser Bedeutung, so dass man die oft vergeblich gemachte Mühe in Kauf nehmen muss. 


\section{Der Aufbau des Dentins.}

Das Dentin, das die Hauptmasse des Zahnes bildet und im Kronenteil von dem Schmelz. im Wurzelteil von dem Zement bedeckt wird, besteht aus einer stark verkalkten Grundsubstanz, die von den Zahnbeinröhrchen durchzogen wird. Die Grundsubstanz hat fibrillüren Charakter und zwar stehen die Fibrillen senkrecht zu den Röhrchen. Der Verlauf der Röhrchen ist ein sehr komplizierter. Nicht allein, dass. jedes Röhrchen zwei grosse Ausbiegungen, welche zusammen eine Sförmige Linie bilden, deren erste Konvexität wurzelwärts, deren zweite kronenwärts gerichtet ist, zeigt; sondern auch noch auf diesem Verlaufe zahlreiche kleine Krümmungen, die bald stärker bald schwächer ausgesprochen sind und fast durchgehends Schraubenwindungen darstellen, aufweisen. Es ist daber nicht wunderzunehmen, dass bei der mikroskopischen Untersuchung dieses Gewebes sich soviel Schwierigkeiten bieten, zumal auch durch die Anordnung der Röhrchen sich schon leicht optische Trugbilder ergeben.

Jedenfalls muss aber festgestellt werden, dass jedes dieser Röhrchen von einer Membran begrenzt: wird, die normalerweise der Wand des Röhrchens fest anliegt und sich bei den meisten Färbemethoden mehr oder weniger stark fürbt. Diese Membran, die leider falscherweise in der Literatur als Neumannsche scheide bezeichnet wird, obwohl sie Kölliker bereits 1852 beschrieben hat, scheint mir identisch mit der von Römer durch Vazerationsversuche dargestellten Membran, del er nur eine falsche Deutung gibt, indem er sie als Begrenzung. der Odontoblastenfortsaitze, die er auf Grund von Querschnittsbildern für Hohlfasern hält. anspricht.

Um diese membranöse Scheide herum ist allerdings. wie Römer sehr richtig beobachtet hat, eine Zone anscheinend nicht stark verkalkter Grundsubstanz, die man aber nur bei gut erhaltenen Querschnittsbildern sehen kann. Im Längsschnitt läsśt dieselbe sich schwer erkennen, wohl durch das Übereinanderlagern verschiedener Zonen, denn man hat bei einem $10 \mu$ Schnitt mehrere Röhrchen übereinander im Schnitt erhalten. Diese Zone, die von jetzt $a b$ als $R$ ömersche bezeichnet werden soll, zeigt sehr schön Fig. 1. Dieselbe ist aber nun bei der Konservierung sehr leicht Schrumpfungen zugänglich, so dass man oft schwer zu deutende Bilder erhält, wie Fig. 2 erkennen 
lässt. Dieses Prăparat zeigt beinahe alle Übergänge, bei a erkennt man ein ganz normales Röhrchen. Der innere Ring stellt die Scheide des Dentinröhrchens dar. Die darum liegende $R$ ö mersche Zone ist hier gar nicht gefärbt.

An vielen Stellen jedoch ist durch Schrumpfungserscheinungen der Grundsubstanz der Römerschen Zone einerseits und durch Quellungserscheinungen der membranösen Scheide des Dentinröhrchens andererseits das Bild so verschoben, dass Stellen wie bei $b$ und $c$ nur auf diese Weise zu deuten sind.

Es besteht also mit Recht die Römersche Ansicht, dass um einen sich intensiv färbenden Ring herum erst eine Zone Grundsubstanz sich befindet, die der der dentinogenen Schicht gleicht, insofern als dieselbe auch auf Farbstoffe wenig, ja bei vielen Färbungen fast gar nicht reagiert.

Der elektiv gefarbte Ring, der die membranöse Auskleidung des Dentinröhrchens darstellt, hat eine lichte Weite von etwas mehr als $1 \mu$. $\mathrm{Zu}$ diesem Messungsresultat kam auch Römer, nur deutet er diesen Ring falsch, indem er in ihm die Begrenzung des Odontoblastenfortsatzes sieht, der sich also seiner Vleinung nach als eine Hohlfaser darstellt.

Dies ist nun keineswegs der Fall, sondern dieser Odontoblastenfortsatz, der leider auch falsch als Tomessche Faser bezeichnet wird, obwohl ihn Neumann zuerst gesehen hat, verläuft als ein massives Gebilde innerhalb dieses bis jetzt beschriebenen Dentinröhrchens, wie Fig. 1 und 2 auch an vielen Stellen erkennen lassen. Nur siebt man ihn nicht immer, ob dies nun daran liegt, dass er sich schwer färbt oder ob er in diesen Fällen herausgefallen ist, lässt sich schwer entscheiden. Jedenfalls in Langsschnittbildern ist er sozusagen an jedem Röhrchen zu sehen und stellt sich immer als ein feiner Faden dar, der aber nicht den ganzen Raum des Röhrchens ausfüllt: Fle ischmann, der auf dieses Verhalten zuerst hingewiesen hat, glaubt, dass dieses Bild durch Schrumpfung des Odontoblastenfortsatzes zustande kommt und dass normalerweise das Röhrchen vollstăndig von der protoplasmatischen Faser erfültt sei.

Dieser Meinung kann ich mich nicht anschliessen. Natürlich soll gerne zugegeben werden, dass derartige Schrumpfungserscheinungen eintreten; aber nicht in dem Maße. Es muss aber auch intra vitam ein Raum zwischen Scheide des Dentinröhrchens 
and Odontoblastenfortsatz bestehen, denn ich konnte ihn von der Zahnhöhle aus durch folgenden Versuch injizieren. Einem frisch extrahierten Zahn wurde nach Trepanation vermittels der Pravazspritze chinesische Tusche sowie auch Asphaltlösung injiziert. der Zahn fixiert und geschnitten. Wie nun Abbildung 3 zeigt, gelang es auf diese Weise selbst noch an der Wurzelspitze diesen Raum zwischen Odontoblastenfortsatz und Dentinröhrchen zu injizieren, also doch ein Beweis, dass wir es hier normalerweise mit einem vorhandenen Raum zu tun haben, der nach theoretischen Erwägungen nichts anderes sein kann, als ein Lymphraum. Dafür spricht die bekannte Tatsache, dass das durch einen Bluterguss verfärbte Dentin, falls die Pulpa am Leben erhalten bleibt, wieder seine normale Farbe erhält.

Querschnittsbilder, die von derartig injizierten /ähnen angefertigt sind, bestätigen, wie Fig. 4 zeigt, nur das bisher Gesagte. Wan erkennt deutlich überall die Scheide des Dentinröhrchens, allerdings ist meist eine Quellung derselben eingetreten auf Kosten der Römerschen Zone, die nur selten gut erhalten geblieben ist.

Man sieht deutlich, dass nur innerhalb der Scheide die T'usche resp. die Asphaltlösung sich niedergeschlagen hat. Die Odontoblastenfortsaitze sind meist nicht zu erkennen, zumal an vielen Stellen die Injektionsmassen das ganze Röhrchen erfüllen.

Nach diesen Ausführungen über den Bau des Zahnbeinröhrchens und seine Beziehungen zum Odontoblastenfortsatz müssen nun noch zwei Gebilde ausserhalb des Dentins an seiner Grenze zur Pulpa Erwähnung finden, da gerade das letztere von ihnen leicht zu Verwechselungen mit nervösen Elementen Anlass geben kann. Es sind dies die bereits 1852 von Kölliker beschriebene Basalmembran und senkrecht $\mathrm{zu}$ ihr verlaufende Bindegewebsfasern, die im engsten Zusammenhange mit derselben stehen.

Was die Basalmembran anbelangt, die als perforierte Membran das Dentin nach der Pulpa abschliesst und von der die Scheiden der Dentinröhrchen ihren Ursprung nehmen, so ist dieselbe für die ganze Auffassung der Genese der Scheiden von fundamentaler Bedeutung. Trotzdem geriet der Befund dieser Membran, die auch als Köllikersches Häutchen bezeichnet wurde, 40 Jahre in Vergessenheit, bis dann Fle is chmann 1905 
ernent darauf hinwies und sie mit dem Namen Lamina terminalis interna belegte. Diese Membran lässt sich durch Mazeration mittels Kalilauge mit den von ihr ausgehenden Scheiden der Dentinröhrchen ziemlich leicht isolieren, sowie auch mit del Bielsehowsky-Methode färben. Sie stellt sich als ein stark fibrilläres Häutchen dar, mitunter von ganz bedeutender Stärke. Von der Pulpa her treten Bindegewebsfasern an dieselbe heran, die für die Histogenese von weitgehendster Bedeutung sind.

Es ist mir gelungen, bei eigentlich für Nervenfärbung verunglïckten $\mathrm{Bi}$ elschowsky-Präparaten Bindegewebsfasern darzustellen, die kolkenzieherartig sich durch die Odontoblastenschicht hindurchschlängeln, sich dann trichterartig auflösen, um mit den Enden in die Lamina terminalis resp. die von ihr ausgehenden Scheiden der Dentinröhrchen überzugehen.

Es unterliegt wohl keinem Zweifel, dass wir es hier mit den in der Literatur von Ebner während der ersten Entwicklung des Dentins beschriebenen Korffschen Fasern zu tun haben, die ja allerdings bereits von $\mathrm{Hoehl}$ beschrieben waren. Zwar wird ja nun hauptsächlich durch von Ebner sowie von anderen Autoren behauptet, dass diese Fasern nach einer gewissen Starke der Dentinbildung $(80 \mu)$ nicht mehr nachweisbar, also etwas vergängliches, embryonales seien und nur Studnicka hat sie vereinzelt in noch alteren Stadien gefunden. Kantorowic $z$ hat sie allerdings an pathologisch veründerten Zăhnen bei der Ersatzdentinbildung auch gesehen und schreibt ihnen eine wichtige Rolle $\mathrm{zu}$, jedoch konnte er dieselben bei ganz normaler Dentinbildung nicht nachweisen, obwohl er ihre Existenz dabei nicht bezweifelt. Dass solche Fasern aber weiterhin in grossem Maße bei ganz normalen Zähnen vorkommen, lässt sich mit der Bielschowsky-Färbung zeigen. Man erhält Bilder wie Fig. 5.

Zusammenfassend gebe ich in Fig. 6 eine schematische Darstellung des Dentins, wie es sich nach den vorstehenden Untersuchungen gestaltet. Man sieht, wie aus dem Bindegewebe der Pulpa die Trichterfasern zur Lamina terminalis interna strömen und wie aus deren Geflecht sich die Wandscheiden der Dentinröhrchen erheben und jene auskleiden. Nach aussen ist jede dieser Scheiden von der Römerschen Substanz umgeben.

In dem Röhrchen liegt, von der Wandscheide durch einen injizierbaren Lymphraum getrennt, der massive Ausläufer der 
Odontoblastenzelle, der Odontoblastenfortsatz. In dem Lymphraum liegt auch ein grosser Teil der Zahnnerven.

\section{Die Innervierung des Dentins.}

Das Dentin muss, weil es sehr empfindlich ist, ausserordentlich reich innerviert sein; über seine Jerven ist deshalb sehr viel gearbeitet worden. Wenn diese Studien bisher zu keinem sicheren Abschluss geführt haben, so ist der Grund darin zu suchen, dass wir bisher keine Methode besassen, die in entkalktem Gewebe mit einiger Sicherheit Nerven nachzuweisen gestattete.

Die ersten Angaben über den Verlaut und die Endigung der Nerven des Zahnes verdanken wir Boll, der im Jahre 1868 eine grundlegende Arbeit über diese Frage veröffentlichte. Boll hat auf Grund von Chromsïureprüparaten festgestellt, dass zahlreiche mit Mark versehene Nervenfasern in Bündel von sechs bis acht meist in der Längsachse des /ahnes aufsteigen allmählich in marklose Fasern übergehen, die besonders unter den Odontoblasten ein dichtes Netzwerk bilden. Vom demselben aus war er imstande, den Verlauf von feinen Fibrillen zwischen den Odontoblasten zu verfolgen, die er für Nervenfasern hält und von denen er annimmt, dass sie in die Zahnbeinröhrchen eintreten, obwohl es ihm nicht gelang, dies nachzuweisen.

Auf diese Ausführungen Bolls wurde in den kommenden Jahren nur immer wieder verwiesen, so von Wedl (1870), Waldeyer (1871), Salter (1874), Tomes (1877), Baume (1885), bis dann von Weil 1888 dieselben stark angegriffen wurden.

Weil sieht absolut keinen Beweis erbracht, dass es sich bei Bolls Fibrillen um Nervenfasern handelt, denn sonst hätte er dieselben bei seinen gut gelungenen Präparaten, an denen er zeigen konnte, dass nach innen von den Odontoblasten sich eine Schicht, die er als Basalschicht der Membrana eboris bezeichnete und die heutzutage den Namen Weilsche Schicht trägt, die aus feinsten durchsichtigen Fibrillen sich zusammensetzt, beobachten müssen. Er konnte jedoch Nervenfasern oder gar Bündel von solchen über die Kortikalschicht der Pulpa hinaus nicht feststellen.

Im Jahre 1891 machte dann Morgenstern seine erste Mitteilung über Nerven des Zahnbeins, denen 1895 und 1896 aus- 
führliche Publikationen über diese Frage folgten. Morgenstern hat mit allen damals zur Verfügung stehenden Methoden versucht, den Verlauf und die Endigung der Zahnnerven klar zu stellen und dafür gebührt ihm doch ein Verdienst. Es ist daher nicht $\mathrm{zu}$ verstehen, dass seine Arbeiten nach kurzen heftigen Angriffen einfach in der Literatur keine Erwăhnung mehr finden, wenn er ja auch sicher bei der Deutung seiner gewonnenen Präparate über das Ziel hinausgeschossen ist. Morgenstern sah in seinen Präparaten Nerven, die teilweise in den Dentinkanălchen (intratubuläre Fasern), sowie solche: die in der Zahnbeingrundsubstanz ausserhalb der Röhrchen (intertubuläre Fasern). Die Endigungen verlegt er an die Schmelzdentingrenze. ja sogar in den Schmelz hinein.

Diese Ausführungen wurden von Röse, Walkhoff und Römer heftig angegriffen und alle drei Autoren behaupteten, dass Morgenstern sich bei der Deutung seiner Bilder' Trugschlüssen hingegeben hat, da sich bei den angewandten Silberfürbemethoden zu leicht auch andere Fasern elektiv färben.

Wenig bekannt in der zahnarztlichen Literatur sind merkwürdigerweise die Untersuchungen von $R$ etzius geworden. Er hat an Mäusezähnen mit der Chromsilbermethode sicher gereigt, dass feine Nervenfibrillen zwischen den Odontoblasten verlaufen und hie und da mit ihnen sowie dem Dentin tangieren. Ein Eintreten in das Dentin konnte er allerdings nicht feststellen.

Diese Befunde konnte $\mathrm{Huber} 1899$ vollauf bestätigen auf Grund von intravitalen Methylenblau-Färbungen bei den Pulpen von Kaninchen, an denen er auch zeigen konnte, dass die Nerven innerhaib der Odontoblastenzone mit verhältnismässig einfachen Nervenendigungen in Gestalt von langen Strablenbündeln von langen varicösen Fibrillen endigen, welche unter den Odontoblasten gefunden werden.

In demselben Jahre berichtet $\mathrm{Römer}$ von seinen ausgedehnten Untersuchungen über diese Frage und glaubt feine Nervenfasern gefunden zu haben, die in die Zahnbeinröhrchen vereinzelt eintreten, während ihres Verlaufes aber im Innern der nur $1 \mu$ dicken Zahnbeinröhrchen nicht erkennbar sind und erst wieder teilweise sichtbar werden in den kolben- und spindelförmigen Erweiterungen der Zahnbeinröhrchen in der untersten 
Schmelzpartie. Dort finden sich kleine rundliche und ovale Körperchen vor, die seiner Ansicht nach als Endkörperchen der sensiblen Nerven angesprochen werden können. Diese Befunde hält Römer 1909 selbst für zweifelhaft, da dieselben auf Grund der vitalen Methylenblau-Färbung gewonnen sind und man sich dabei zu leicht Täuschungen hingibt. Theoretisch hält er es für keineswegs zweifelhaft, nur musste noch der histologische Nachweis erfolgen.

Da nun die Frage nach den Nerven des Dentins bis vor kurzem im wesentlichen, wie man sieht, eine offene geblieben war, die Emptindlichkeit desselben nicht geleugnet werden konnte, so hat man sich vielfach auch nach einer anderen Lösung des Problems umgesehen.

Einige Autoren (Colemann, Hopewell, Smith, Ligros, Magitît, Bödecker) halten es tür sehr möglich, dass die weithin in das Dentin ausstrablenden Odontoblastenfortsïtze die Trïger der Sensibilitüt seien. Bis an die Odontoblasten sind ja von Retzius und $\mathrm{Huber}$ Nerven verfolgt und auch von früheren Autoren hat niemand das Vorkommen von Nerven bis an das Zahnbein bestritten. Würe dem aber so, dann hätte man bei dem ungeheueren Reichtum von Pulpanerven irgendwelche GeHechte $\mathrm{um}$ die Odontoblasten $z u$ erwarten. Weil dem nicht so ist, zahlreiche Nerven an der Grenze des Dentins geradezu verschwinden, ist man weiter bestrebt, nachzusehen, wohin diese gelangen. Meine eigenen Untersuchungen, über die im folgenden berichtet werden soll, haben bereits im April 1912 zweifellos Nerven in den Zahnbeinröhrchen auffinden lassen. Nerven, die ich damals und später wiederholt mit anatomischen Autoritäten, die das Neurologische Institut besichtigten, am Präparat diskutieren konnte. Ich darf diese Gelegenheit benutzen, den Herren Nageotte, Erick Müller, Holmgren, Waldeyer und O. Schulze meinen besten Dank zu sagen.

Gleichzeitig mit mir haben Mummery und auch Dependorf auf dem gleichen Gebiet gearbeitet. Dezember 1912 hat Mummery eine Arbeit über Nerven im Zahnbein veröffentlicht, die er mittels des B e ckwirthschen Chlorgold-Verfahrens zur Darstellung brachte. Er zeigte, dass die Nervenbündel sich pinselförmig in Nervenfibrillen auflösen, die schliesslich nach teilweisem seitlichen Ausbiegen in die Zahnbeinröhrchen eintreten. 
Schliesslich ist noch im Juni dieses Jahres eine Arbeit von Dependorf erschienen, der auch Nerven im Zahnbein nachweisen konnte und zwar mittels Farbungen nach der Methode von Löwitz, Bielschowsky und Held. Auf diese Arbeit werde ich bei meinen eigenen Befunden noch des öfteren zu sprechen kommen, um so mehr als ich Gelegenheit hatte, die Präparate selbst einzusehen und mich von ihrer zweifelsohne richtigen Deutung zu überzeugen.

Ich selbst habe mit der oben erwähnten BielschowskyTechnik gearbeitet. Als Nervenfasern wurden nur solche Gebilde angesprochen, die sich als glattrandiger gestreckter Faden. an dem gelegentlich kleine Varikositäten sind, von der immer mehr oder weniger gewellten und unregelmässig gerandeten Bindegewebsfaser wohl unterscheiden liessen. Wenn die letzteren gelegentlich sich ebenfalls mit Silber intensiv imprägnielt hatten, war die Unterscheidung so schwer, dass von der Benutzung solcher Präparate abgesehen werden musste. Aber das Giuick wollte es öfter, dass sich nur Nervenfasern imprïgnierten, das Bindegewebe sich nur schwach und viel heller färbte.

An solchen Präparaten war es dann auch oft möglich, das, was ich für Nerven halte, pulpawarts direktbis in die markhaltigen Nervenfasern zu verfolgen. Damit ist der sichere Beweis für. die Richtigkeit meiner A uffassung gegeben.

Fig. 7 Jässt deutlich erkennen, dass von den parallel zu dem Dentin verlaufenden Hauptnervenstämmen aus feine marklose Fasern horizontal durch die Odontoblastenschicht bindurchtreten und in die sogenannte dentinogene Schicht eindringen: in derselben meist in den präformierten Dentinröhrchen neben dem Odontoblasten verlaufen, also in dem bereits beschriebenen Lymphraum. Jedoch sind die Fasern nur immer bis zur nicht stark verkalkten Schicht zu verfolgen, ein Umstand, der leicht erklärt wird durch das übermässige Färben dieser Zone. In diesem so tief gefärbten Gewebe lässt sich natürlich kein solch feiner schwarzer Faden, wie sich der Nervenfaden charakterisiert, mehr erkennen. Andererseits ist es aber auch nicht möglich, weniger stark zu färben, da meist erst nach dem achten bis 
Untersachungen über den Bau und die Innervierung des Dentins. 317

zehnten Vale wiederholten Färben sich die feinsten I asern erkennen lassen.

Es ist somit natürlich auch gar nichts über die Zahl der eintretenden Fasern auszusagen, denn wenn man dieselben auch nicht sieht. so ist es kein Beweis, dass sie etwa nicht vorhanden sind. Es ist gar nicht abzustreiten, ob nicht vielleicht in jedes Dentinröhrchen eine solche Faser eintritt, obwohl dies: nicht anzunebmen ist und zwar aus gleich zu besprechenden Gründen.

Wie nämlich schon Fig. 7 an einer Stelle und Fig. 8 an mehreren stellen zeigt, verlaufen nicht alle Nervenfasern, die durch die Odontoblastenschicht hindurchgetreten sind, in den Lymphräumen der Dentinröhrchen. sondern auch quer durch die Grundsubstanz, ja manche biegen sogar direkt in ihr um. Diese Befunde stimmen nun vollkommen mit denen von bependort ïberein. Derselbe hat solche Querfasern allerdings, wie ich mich an seinen eigenen Präparaten überzeugen konnte, sogar überall in der stark verkalkten Grundsubstanz gefunden. was mir bis jetzt noch nicht möglich war. Nur in der dentinogenen schicht habe ich Bilder, wie er sie zeigen konnte, anch gesehen. Gründe dafür. anzugeben, warum es mir nicht gelang, in stark verkalktel Grundsubstanz solche Fasern darzustellen, ist einfach unmöglich. denn warum sich bei diesen Silbermethoden das eine oder das andere Mal die Nervenfasern sich nicht oder wenigstens nur gan\% wenig fürben, lässt sich nicht sagen. So zeigen Fig. !) und 1! beispielsweise zwei Nervenfasern, die ganz besonders stark exscheinen, centripedal sich an den Hauptstamm verfolgen lassen. aber in beiden Präparaten die einzigen Fasern sind, die sich in der Odontoblasten- sowie dentinogenen Schicht tingiert haben. Es ist dies allerdings verwunderlich, aber in der Nervenhistologie eine bekannte Erscheinung.

Es steht somit also sicher fest, dass das Dentin mit Nerven versorgt wird und findet seine Sensibilität eine leichte Erklärung. Ja es zeigt sich sogar, dass die Morgensternsche Anschauung. es gäbe zwei Systeme von Nervenfasern, richtig ist. Ob allerdings Morgenstern dieselben bereits gesehen hat, wenigstens die wirklichen, bleibt dahin gestellt. Jedenfalls muss man nunmehr zwischen Nervenfasern unterscheiden, die in der Grundsubstanz des Dentins verlaufen, gegenüber solchen, die in die 
IJentinröhrchen eintreten und dort in dem von mir jetzt aufgefundenen Lymphraum verlaufen.

Es bleibt nunmehr nur noch die Frage offen, wo haben wir die Endigungen zu suchen und zwar von beiden Systemen. Bleiben die ersteren in der Grundsubstanz und endigen in derselben und verlaufen die letzteren durch das ganze Röbrchen und treten etwa gar in den Schmelz ein. Das sind beides offene Fragen, deren Lösung einzig und allein von der mikroskopischen Technik abhăngt. Sollte diese sich für derartig schwer zu behandelnde Objekte bald weiter vervollkommnen, so werden auch diese Fragen sicher bald einer wirklichen Lösung entgegengebracht.

Jedenfalls ist von grossem Werte, dass nunmehr alle Theorien über die Sensibilitat des Zahnbeins hinfallig sind, nachdem der sichere Beweis der Nervenversorgung desselben erbracht ist. Somit kann natürlich den Odontoblasten bei der Bildung des Dentins eine grössere Rolle wieder zugesprochen werden, wie weit jedoch, darüber müssen erst weitere Arbeiten Aufschluss geben, denn die mit Nervenfasern so leicht zu verwechselnden Trichterfasern ( $\mathrm{K}$ or $\mathrm{ff}$ sche), deren Nachweis mir auch bei ausgebildeten Zühnen gelungen ist, sprechen sicher bei diesen Bildungsprozessen eine nicht wenig bedeutende Rolle.

Herrn Prof. Edinger, Herrn Dr. Doinikow und Herrn Dr. Stendell, die laufend meine Präparate kontrolliert haben, danke ich für ihr freundliches Interesse.

\section{Literaturverzeichnis.}

Boll, F.: Untersuchungen über die Zahnpulpa. Arch.f. mikr. Anat., IV, 1868.

Depend orf: Ergebnisse eigener Untersuchungen über Innervierung des menschlichen Zahnes mit Berücksichtigung der Hartsubstanzen. Deutsch. Monatsschr. f. Zahnheilk., 31. Jahrg., H. 6, 1913.

v. Ebner: Über scheinbare und wirkliche Radiärfasern des Zahnbeines. Anat. Anz., Bd. 34.

Fleischmann, L.: Histologie und Histogenese der Zähne. Ergebn. d. ges. Zahnheilk., I. Jahrg., H. 1, 1911.

Huber, G. C.: Die Innervation der Zahnpulpa. Correspondenzbl. f. Zahnärzte, Bd. XXVIII, 1899, H. 1.

Kantorowicz, A.: Zur Histogenese des Dentins, insbesondere des Ersatzdentins. Deutsche Monatsschr. f. Zahnheilk., 1910, H. 8. 
v. Korff: Die Entwicklung der Zahnbeingrandsubstanz der Säugetiere. Arch. f. mikr. Anat. u. Entwicklungsgesch., Bd. 67.

Derselbe: Entgegnung auf die v. $\mathrm{E}$ bnersche Abhandlung, Über scheinbare und wirkliche Radiärfasern des Zahnbeins". Anat. Anz., Bd. 35.

Morgenstern, M. : Vorläufige Mitteilung von Nerven im Dentin. Deutsche Monatsschr. f. Zahnheilk., 1892, H. :0.

Derselbe: Vorkommen von Nerven in harten Zahnsubstanzen und eine Methode, sie aufzufinden. Deutsche Monatsschr. f. Zahnheilk., 1895, H. 3.

Derselbe: Über die Innervation des Zahnbeins. Arch. f. Anat. u. Phys., 1896.

Derselbe: Der gegenwärtige Standpunkt unserer Kenntnis der Zahnbeinnerven. Correspondenzbl. f. Zahnärzte, 1899, H. 2.

Mummery, H.: A short Supplementary Note on the Nerves of the Dentine. Proceedings of the Royal Society of Medizine, 1912, Dezember.

Reich, P.: Das irreguläre Dentin der Gebrauchsperiode. Marburg 1906.

Retzius: Zur Kenntnis der Nervenendigungen in den Zähnen. Biolog. Untersuchungen, 1892.

Derselbe: Über die Nervenendigungen in den Zähnen von Amphibien. Biolog. Untersuchungen, 1893.

Derselbe: Zur Kenntnis der Endigungsweise der Nerven in den Zähnen der Süugetiere. Biolog. Untersuchungen, 1894.

Römer, O.: Über Sensibilität des Zahnbeins. Deutsche Monatsschr. f. Zahnheilk, 1899 , H. 9.

Derselbe: Über die Pathologie der Zahnpulpa. Verh. d. V. Intern. Zahnärztl. Kongresses, Berlin 1909.

Derselbe: Veründerungen der Dentinkanälchen bei der Zahnkaries. Verh. d. V. Intern. Zahnärztl. Kongresses, Berlin 1909.

Derseibe: Atlas der pathologisch-anatomischen Veränderungen der Zahnpulpa nebst Beiträgen zur normalen Anatomie von Zahnbein und Pulpa beim Menschen. Freiburg 1909.

Rüse: Über Nervenendigungen in den Zähnen. Deutsche Monatsschr. f. Zahnheilk., 1893, H. 2.

Studnička: Die radialen Fibrillensysteme bei der Dentinentwicklung und im entwickelten Dentin der Säugetiere. Anat. Anz., Bd. 30.

Walkhoff: Abwehr des Herrn Morgenstern. Deutsche Monatsschr. f. Zahnheilk., 1902, H. 9.

Weil: Zur Histologie der Zahnpulpa. Deutsche Monatsschr. f. Zahnheilk. 1887, H. $9 ; 1888$, H. 1. 


\section{Erklärung der Abbildungen auf Tafel XIII und XIV.}

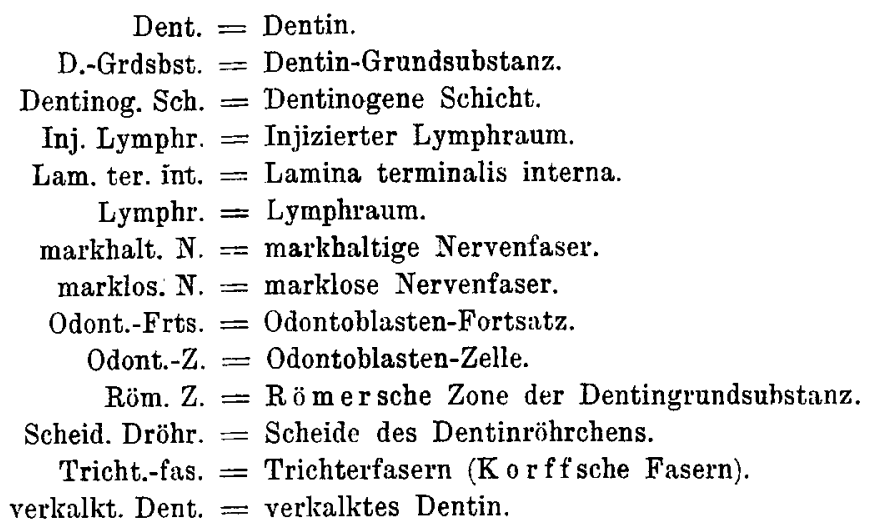

Fig. 1. Querschnitt durch das Dentin eines Molaren. Gut erhalten. Biels c h ow sky - Färbung.

Fig. 2. Querschnitt durch das Dentin eines Molaren. Schlecht erhalten. Hämatoxylin-Färbung.

Fig. 3. Längsschnitt durch das Dentin eines mit Tusche injizierten Eckzahnes. Hämatoxylin-Färbung.

Fig. 4. Querschnitt durch das Dentin eines mit Asphaltlösung injizierten Molaren. Hämatoxylin-Färbung.

Fig. j. Längsschnitt durch das Dentin einrs Prämolaren. Bies chowskyFärbung.

Fig. 6. Schematische Darstellung des Baues eines Dentinröhrchens.

Fig. 7. Lüngsschnitt durch einen Prämolaren. Dentin mit Pulpa. Biels c ho w s ky - Fürbung.

Fig. 8. Längsschnitt eines Eckzahnes. Dentin mit Pulpa. Bielschowsky Färbung

Fig. 9. Längssclnitt eines Eckzahnes. Dentin mit Pulpa. Bielschowsky Färbung.

Fig. 10. Längsschnitt eines Eckzahnes. Dentin mit Pulpa. BielschowskyFärbung. 


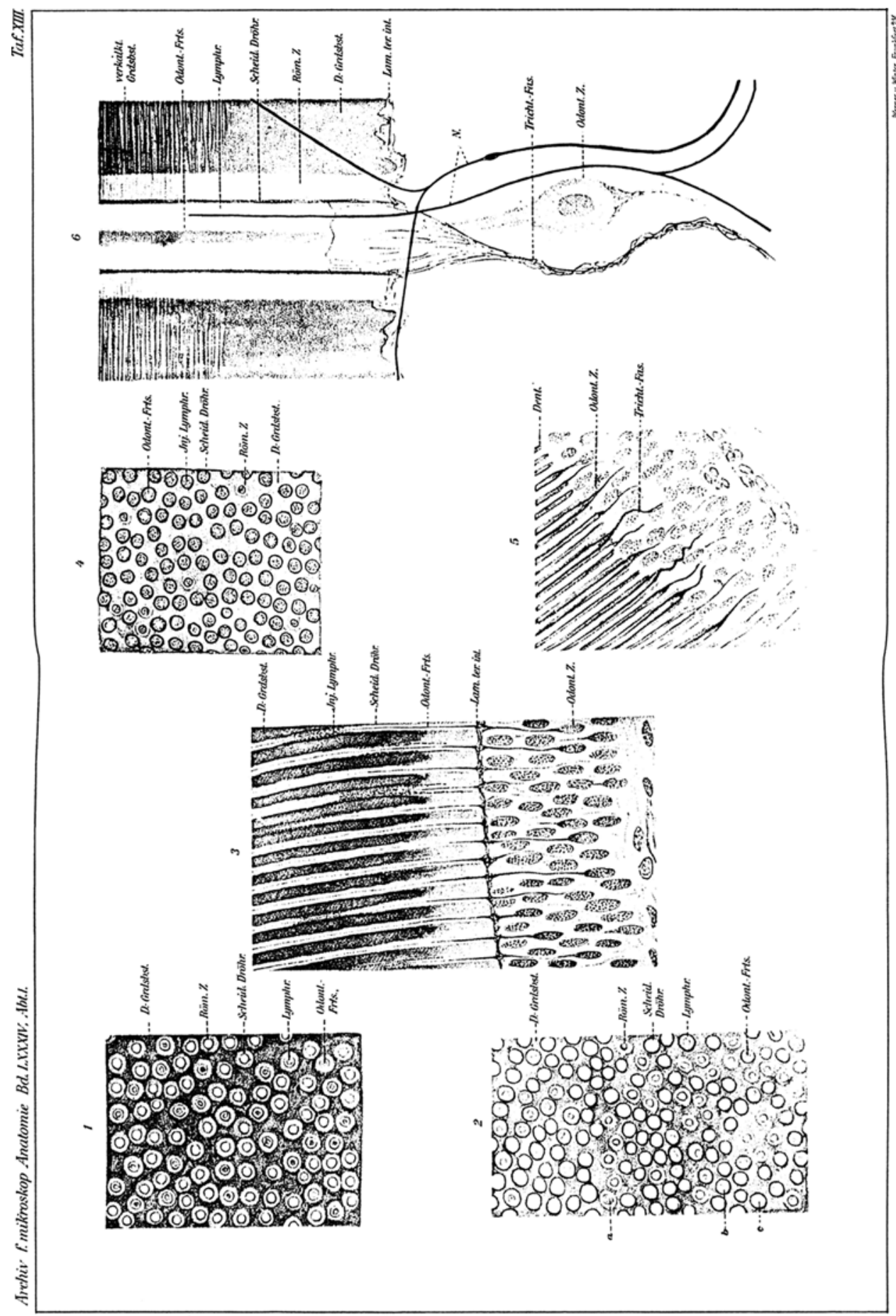



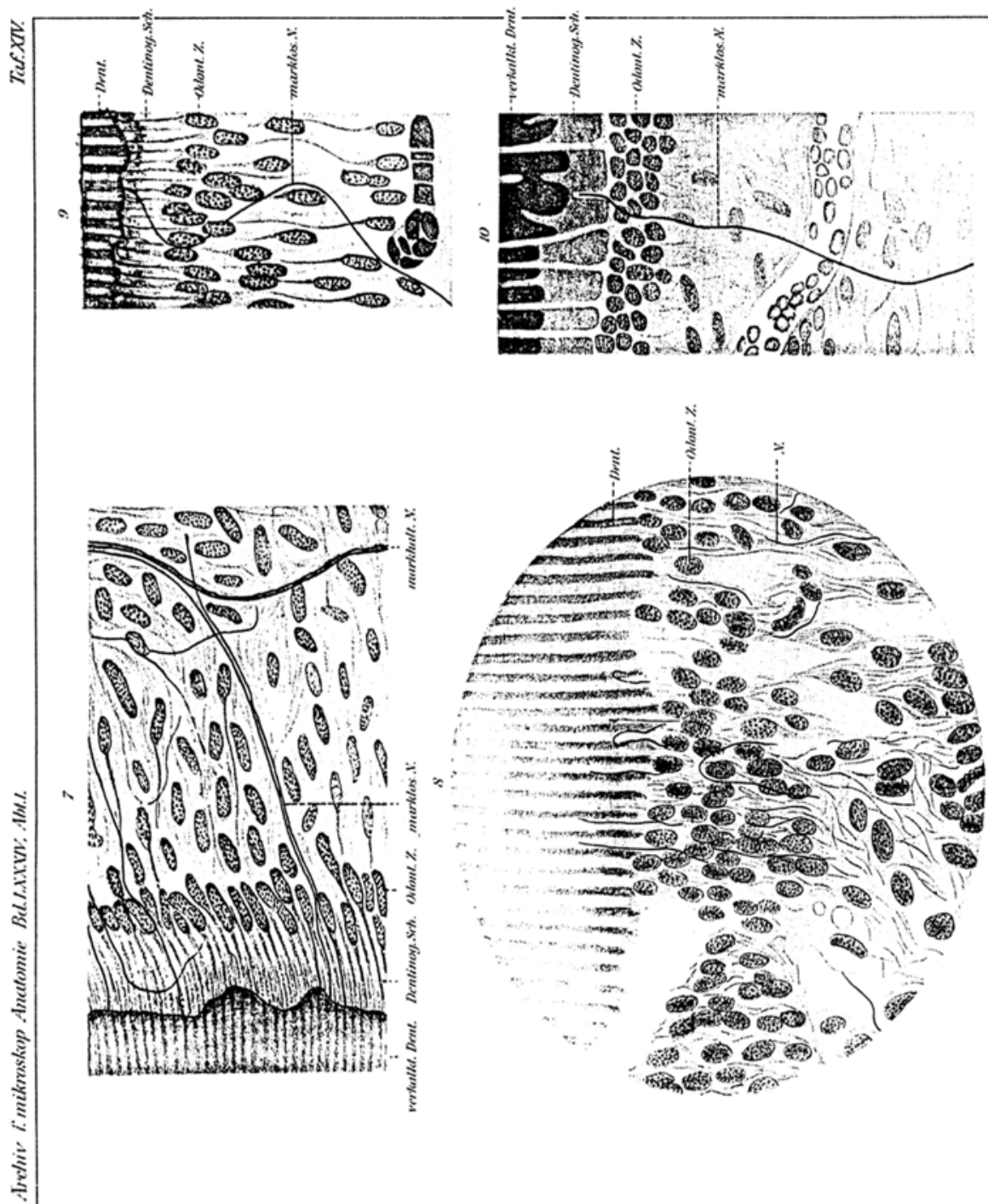